Meta

Journal des traducteurs

Translators' Journal

\title{
Problèmes terminologiques des pluies acides : pour une socio-terminologie
}

\section{Yves Gambier}

Volume 32, numéro 3, septembre 1987

La fertilisation terminologique dans les langues romanes

URI : https://id.erudit.org/iderudit/002791ar

DOI : https://doi.org/10.7202/002791ar

Aller au sommaire du numéro

Éditeur(s)

Les Presses de l'Université de Montréal

ISSN

0026-0452 (imprimé)

1492-1421 (numérique)

Découvrir la revue

Citer cet article

Gambier, Y. (1987). Problèmes terminologiques des pluies acides : pour une socio-terminologie. Meta, 32(3), 314-320. https://doi.org/10.7202/002791ar d'utilisation que vous pouvez consulter en ligne. 


\title{
PROBLÈMES TERMINOLOGIQUES DES PLUIES ACIDES : POUR UNE SOCIO-TERMINOLOGIE
}

\author{
Yves GAMBIER \\ Université de Turku, Finlande
}

Les modes de production et la circulation des savoirs et des biens sont tels aujourd'hui qu'il est quasiment impossible de circonscrire de manière catégorique un " domaine " de connaissance, de fabrication : la compétition internationale, l'informatisation, le rôle social et le fonctionnement des médias sont parmi les facteurs qui poussent à l'intégration de tous les domaines. Toutefois, ces interférences entre des champs naguère séparés n'excluent pas la spécialisation. Les flux d'échange s'accélèrent en partie aussi à cause de la division du travail. Ce paradoxe est l'une des bases de notre économie ; il est également une des bases de développement de la recherche : celle-ci est de plus en plus pointue dans certains cas tout en appelant à l'interdisciplinarité et elle devient vite l'objet de discours publics, confrontée à d'autres secteurs, à des besoins d'application. C'est le cas, par exemple en biologie (découvertes génétiques et problèmes de la reproduction). C'est le cas des précipitations acides (dites encore dépôts ou pluies acides). Quelles conclusions doit-on tirer pour la terminologie? Dans la propagation des savoirs et des savoir-faire, que devient la terminologie?

Trois caractéristiques des retombées acides - qui polluent par la pluie, la neige, la grêle, les brumes, les gaz, les poussières, c'est-à-dire par des dépôts humides ou secs influencent leur terminologie :

- elles ont des effets variables, plus ou moins directs, sur les lacs, les cours d'eau, les forêts, certaines cultures... suivant la nature de l'écosystème touché, sans parler des effets sur les constructions, la santé... : l'acidité n'est pas une valeur absolue ${ }^{1}$; en chimie, est " acide " ce qui est inférieur à un pH de 7 ; avec les dépôts acides, l'acidité oscille entre au-dessous de 5,6 (pluie acide) et au-dessous de 5,5 (lac acide); plexe $^{2}$;

- les retombées acides sont le résultat d'un processus physicochimique com-

- elles sont une partie des pollutions (atmosphériques, des eaux...), elles ont la particularité aussi d'être transfrontières.

Cela a plusieurs implications : le "domaine " des dépôts acides fait appel à diverses spécialités (chimie, météorologie, géologie, sylviculture, biologie, mathématiques, droit, étude des matériaux...); il est à la fois objet de recherche scientifique avec encore nombre d'incertitudes et d'inconnues, objet d'interventions juridiques (définition de normes, de moyens de protection) et objet de prises de position publiques d'écologistes, de politiciens, d'industriels... On ne saurait sous-estimer le fait que le sujet est apparu d'abord sous la pression de l'opinion, de militants : il est lié au développement de certains mythes quant à l'environnement " naturel ", au développement de la pollution comme problème de société et de technique, renforcé par les médias. 


\section{CORPUS ET TYPOLOGIE}

\section{CORPUS}

La collecte des termes est le résultat de trois mois de travail à la Direction de la terminologie du Parlement européen (PE) - Luxembourg, mai-juillet 1983³. Le corpus de traitement a été constitué d'ouvrages scientifiques, de rapports, d'actes de conférences (de 1972 à 1982), de publications officielles - parfois bilingues (anglais-français) : traités, accords, résolutions, conventions - d'origine variée : groupe de chercheurs, Conseil de l'Europe, OCDE, PE, CEE, ONU-CE, Comecon, etc. On y a ajouté une certaine masse d'articles d'une trentaine de périodiques dont Environmental Policy and Law, Europe-Environnement, Observateur de l'OCDE, Environnement, Ambio, Ends Report... ainsi que des dictionnaires, vocabulaires et glossaires sur la protection de l'environnement, la pollution de l'air.

Nous avons ainsi relevé plus de 400 termes français touchant les dépôts acides, d'occurrence plus ou moins fréquente ; tous ne sont pas des néologismes. On a complété ces sources par le No 40-41, 1985 de Néologie en marche (coordonné par D. Rivard), fruit d'une collaboration entre le Secrétariat d'État d'Ottawa et l'Office de la langue française (Québec) : dans ce cahier, une centaine de notions a été traitée pour un total de 197 termes. Ceux-ci recoupent en grande partie nos 400 dénominations - même s'ils ont été pris dans un fonds documentaire plus nord-américain qu'européen. Plusieurs remarques s'imposent quant à la documentation sur les dépôts acides :

- il n'existe à l'heure actuelle aucun dictionnaire, aucune monographie en français langue source, qui leur seraient consacrés exclusivement ;

- il existe une assez forte proportion de références " grand public ", de caractère polémique plus que scientifique (pour la défense de l'environnement ; contre l'inertie des gouvernements ; controverses sur les mesures prises ; les moyens de lutte à mettre en cuvre...);

- il faut puiser à des sources interdisciplinaires et mixtes (documents de recherche et de vulgarisation).

Hybride dans ses origines, diffusée rapidement et pour des supports variés, la terminologie des "pluies acides" soulève donc un certain nombre de problèmes que nous allons traiter en esquissant d'abord une typologie.

\section{ESSAI DE TYPOLOGIE DES TERMES}

\section{a) Transfert de termes et néologismes sémantiques}

On distinguera dans cette catégorie cinq sous-groupes :

1. Le domaine des dépôts acides est un champ de composite : il a emprunté un certain nombre de termes à d'autres domaines, avec glissement de sens plus ou moins prononcé. Par ex. :

- de la climatologie : bassin d'air, neige propre/normale, pluie propre/ ordinaire/pure, précipitation naturelle...

- de la météorologie : brouillard, lavage, période sèche...

- des mathématiques : modèle Lagrangien, méthode de Löblich...

- de la chimie : absorptiométrie, acidification naturelle, acides (nitrique, sulfurique), aérosols, alcalinité, lac non acide, dioxyde, anydride (carbonique, sulfureux...), calcaire, calcite, colorimétrie, métaux lourds, oxydants...

Ces signifiants subissent des transformations sémantiques lentes, légères et fragiles : selon les contextes, ils peuvent garder plus ou moins leur sens original. Ce flottement rend difficiles leur collecte et leur traitement. En fait, il y a ici reflet des difficultés à définir le phénomène "pluies acides": on a vite dénoncé les effets, on a vite cherché à 
départager les responsabilités et les coûts... sans savoir exactement comment fixer les origines, les causes, comment mesurer les impacts... par rapport à cette réalité qu'on croyait connaître : la pluie normale, propre, pure, ordinaire. Sous la pression publique, très vite, on a voulu analyser la composition chimique des dépôts, les processus physiques et météorologiques suivis dans l'atmosphère, les traces laissées dans divers écosystèmes. D'où le recours à des termes abstraits, de description plutôt qu'à des termes désignant un appareillage, des instruments...

2. Certains termes connaissent une variation de genre (sing.-pluriel) qui est hésitation ou nuance de sens. Par ex. : dépôt (action de se déposer) - dépôts (matières déposées) ; pluie(s) acide(s) (singulier calquée de l'anglais ?) ; précipitation(s)...

3. D'autres termes sont le résultat d'une conversion (changement de catégorie grammaticale). Par ex. : local-ement, global-ement, régional-ement (adj. $\rightarrow$ adv.). On notera incidemment ici la faible quantité de verbes liés aux dépôts acides ; on a : tamponner, chauler, lessiver, désulfurer...

4. Il existe quelques homonymes : par ex. retombées acides : 1) matières polluantes acides d'origine atmosphérique, qui se sont déposées - synonyme alors de dépôt acide ; le sens 2) est synonyme de pollution acide, phénomène d'altération écologique. Autres ex. : lac acide, lac acidifié, lavage... Deux sigles ont une dimension homonymique : TAC ( 1 : taux d'appauvrissement en cations ; 2 : titre alcalimétrique complet), TGDPA ( 1 : transport à grande distance des polluants atmosphériques ; 2 : transport à grande distance de la pollution atmosphérique).

5. Enfin, il existe les nombreux cas de quasi synonymie auxquels on peut ajouter des termes dont les sens tendent à se confondre de plus en plus. Par ex. : charge acide/ dépôt acide, apport/dépôt, lavage/lessivage, potentiel de neutralisation de l'acide/ capacité de neutralisation de ou des acides/pouvoir neutralisant des acides, précipitation acide/pluie acide (précipitation peut comprendre aussi en fait la neige, la grêle, la brume...) - Voir aussi infra $\mathrm{C} 1$ les synonymes indiqués après une barre oblique (ex. dépôt brut/global).

Ces termes voisins s'expliquent en partie parce que le phénomène décrit est complexe et en cours d'étude.

On signalera ici les termes qui fonctionnent en série binaire ou ternaire (opposition significative) : par ex. acidification naturelle anthropogène, les deux types s'opposant eux-mêmes à acidification expérimentale; retombées humides sèches; source éloignée/ distante étendue/diffuse locale ; source étendue ponctuelle ; source fixe mobile ; concentration relative absolue... L'interdépendance de local régional global, de courte moyenne grande distance permet, dans la modélisation du transport atmosphérique des polluants, une délimitation sémantique précise puisque : local = distance inférieure à $100 \mathrm{~km}$ ou $48 \mathrm{~h}$ de transport atmosphérique, régional - distance supérieure à quelques centaines de $\mathrm{km}$ ou à $48 \mathrm{~h}$, global = distance supérieure à une dizaine de milliers de $\mathrm{km}$ ou à $72 \mathrm{~h}$; au-delà des mesures physiques, ces termes désignant surtout des responsabilités territoriales que recouvrent aussi parfois les notions de faible, moyenne ... distance.

b) Les recherches sur les précipitations acides ont été souvent décrites en anglais - directement (aux É.-U., au Canada) ou non : ainsi les pays nordiques, certaines organisations et associations internationales rédigent ou traduisent leurs textes en anglais. Malgré tout, la diffusion en français (Québec ; organisations européennes) connaît assez peu de calques; on a par ex. anthropogénique (anthropogenic acidification) pour anthropique, bassin calibré (calibrated watershed), capacité d'absorption des protons (CAP) d'ailleurs attesté seulement dans les traductions, stress acide - concurrencé par agression/pression acide, excessif (in excess), épisodicité (episodicity) pour périodicité, source 
stationnaire pour source fixe... On note un faux-ami d'usage fréquent : global pour mondial. Les emprunts enfin sont plutôt rares : ex. rain-out, parfois rendu par piégeage...

\section{c) Composés attestés et néologismes formels}

Il est assez difficile d'évaluer le degré de lexicalisation, la fréquence d'occurrence de tel ou tel terme. Pourtant on peut constater que les composés qui reviennent souvent suivent un nombre restreint de modèles ( 3 principaux) :

1. Dérivés syntagmatiques constitués d'un nom + adjectif ou participe (passé, présent) dans l'ordre déterminé-déterminant. Par ex. acidification artificielle, hydrique, naturelle; agents photochimiques; alcalinité antérieure/initiale; anion majeur/ dominant/principal ; charge acide ; charge atmosphérique ; choc acide ; concentration ambiante/moyenne ; conversion chimique ; cendres volantes ; dépôts acides, humides, secs ; dépôt brut/global, total ; émissions fugitives, globales ; importateur net ; neige acide, noire ; cour mouillé (des sapins) ; polluant précurseur, primaire, secondaire ; pluie acide similée ; source majeure ; mesures multilatérales, primaires, secondaires ; nuisances transrégionales ; pays concerné, exposé ; dépoussiérage intensif ; effet chronique, dommageable, nocif ; émetteurs fixes, etc.

Comme très souvent dans les terminologies, la valeur des adjectifs tend à se distinguer de leur sens courant, pour acquérir une précision plus grande : ainsi la "source linéaire " (ou "sources alignées") désigne la source d'émissions polluantes, constituée de plusieurs sources, de taux d'émission assez comparable et se suivant dans l'espace ; ainsi la "source majeure" (fixe ou mobile, ponctuelle ou étendue) est une source qui rejette plus de 100 tonnes de polluants atmosphériques par année.

2. Composés avec joncteurs

- nom + de + nom : ex. bassin d'air ; charge de polluants ; dépôt d'acides ; niveau/degré d'acidité ; compteur de poussières ; indice de saturation calcique ; plafond d'émission ; stress d'acidité ; station d'échantillonnage ; taux d'appauvrissement en cations, de conversion, de dépôts, d'émission ; usine d'acide ; vitesse de corrosion/de dépôt ; installation d'épuration de gaz ; perte de fertilité du sol ; pays d'origine ; phase d'accumulation, de déstabilisation, de dépérissement, de transition...

- nom + à/en/sur/par + nom : ex. collecteur à deux augets, transport à courte distance ; charbon à faible teneur en soufre; teneur en cendres; particules en suspension ; expérience en chambre à smog; combustion en/par/sur lit fluidịsé ; modèle par trajectoire ; détecteur d'/par ionisation à la flamme...

- Par rapport à ces lexies par subordination, les composés par juxtaposition sont très peu nombreux (nom $+\mathrm{O}=$ nom) : ex. effet tampon, pouvoir tampon, valeurs guides, valeurs limites...

3. La dernière catégorie de termes qu'on peut établir, rassemble :

- les sigles, ex : ARGA, CAP, CEC, DGC, EMEP, FID, FOR, ISC, PNA, PTF, TAC, TDC, TGDPA, TGS, SCR;

- et les éponymes, ex. : méthode colorimétrique Griess-Saltzman, méthode Saltzman en continu, méthode Jacobs-Hochheiser, modèle de dispersion Lagrangien, méthode de Löblisch...

Que conclure?

Le microchamp sémantique des dépôts acides est en évolution, ouvert - au contraire par ex. du vocabulaire de l'anatomie, de la faune ou d'autres considérés comme relativement stabilisés. Il est un ensemble de désignations en nombre assez limité, dominé par des formes composées (cf. $\mathrm{Cl}$ et 2) et des termes transfuges de domaines connexes (cf. a.1). Enfin il éclaire assez nettement les corrélations possibles entre des don- 
nées terminologiques et des phénomènes sociaux - dans de cas, la mise en place d'un savoir scientifique, issu d'une inquiétude collective et appliqué à résorber, dépasser cette inquiétude.

\section{TERMINOLOGIE EN CONTEXTE}

Trop peu souvent, les termes sont vus dans leur utilisation discursive. On peut se demander pourtant si leur traitement dans divers types de texte n'est pas sans impact sur leur détermination, leur définition. Dans la plupart des méthodologies, on suggère aux terminographes de puiser aux documents produits et utilisés par les spécialistes. Ce qui implique, naïvement, un domaine assez délimité auquel se rattache un corps d'experts. Que se passe-t-il quand les sources de la terminologie ont des enjeux différents, comme dans notre cas? On l'a vu, les "pluies acides " sont la préoccupation de chimistes, de défenseurs de l'environnement, de fonctionnaires, de juristes... Leur manière par ex. d'établir les causes du phénomène n'est pas la même - d'où par ex. les polémiques entre l'administration Reagan et le gouvernement canadien sur la pollution atmosphérique du Nord-Est américain et le pourtour des Grands Lacs, d'où les polémiques entre les Européens pour réduire les émissions de soufre et d'azote, de carbone, en recourant respectivement aux combustibles propres et à l'essence sans plomb, d'où les hésitations dans la mise en place des projets de coopération (pour la mesure, la surveillance, la prévention...).

Ici la synonymie (équivalents plus ou moins approximatifs) apparaît dans toute sa fonction : elle n'est pas une concurrence formelle de termes. Sémantiquement, "pluies acides ", "précipitations acides", "polluants atmosphériques", " pollution atmosphérique transfrontière/transfrontalière à longue distance » peuvent se recouper. Seulement ils ne sont pas utilisés dans les mêmes conditions - les rapports, conventions, protocoles mettent davantage en évidence les deux derniers termes (cf. documents de la conférence de Stockholm : 1972, de l'OCDE : 1974-81, de la Commission économique pour l'Europe de l'ONU : 1979 et 1985, de la CEE : 1978, du Conseil de l'Europe : 1979, du Parlement européen : 1983...) tandis que la grande presse (le Monde, l'Express, 30 jours d'Europe, le Courrier de l'Unesco...), les revues dites de vulgarisation (Sciences et Avenir, la Recherche, Québec Science, Observateur de l'OCDE...), les notes officielles d'information (services d'ambassade, ministères de l'Environnement, communiqués du Conseil des Ministres...) - entre 1975 et aujourd'hui, parlent délibérément de "pluies acides " (par ex. dans les titres : "Les pluies acides : holocauste écologique? ", "Odyssée des pluies acides", "Le péril acide", "Du vinaigre tombe du ciel : de l'acide dans les pluies", "Le monde malade de l'acide ", "Acidus sulfuricus", "L'acide venu du ciel "...). La différence est dans le type et la visée de communication dans un cas, le discours prétend à l'objectivité et tend à dépassionner la question pour définir des responsabilités (visée plutôt juridique); dans l'autre, il y a description (des effets surtout) et polémique (dénonciation ou effort pour désamorcer ces dénonciations) - il faut noter cependant que, dans ce groupe, "pluies acides » reste souvent entre guillemets.

Cet aspect de l'emploi des termes mériterait d'être approfondi, par ex. par l'analyse des manières d'introduire les termes ressentis comme spécifiques, néologiques, de formuler les définitions, les nuances de sens. Dans les textes du premier groupe, on a ainsi le souci explicite de définir (paragraphes pour dire ce qu'on entend par "pollution de l'air ", "particules en suspension »...); dans le second groupe, on s'appuie beaucoup sur de l'implicite, du non-dit - sauf pour les notions scientifiques (ainsi " $\mathrm{pH}$ » est souvent précisé mais pas "dépérissement ", " oxyde de soufre " mais pas "lac mort ", "cheminée géante "...). On ne peut cependant pas affirmer que l'emploi des définitions est in- 
versement proportionnel à la spécialisation du document (plus un texte serait spécialisé, moins il définirait).

En fait, il faut ici se reposer la question du « domaine » : les précipitations acides constituent-elles en soi un domaine de connaissances et de savoir-faire, une terminologie? Peut-on même parler de spécialistes de ces précipitations? Il semble que non : - l'objet "pluies acides » reste équivoque, mal connu, sous-ensemble peut-être de la pollution (atmosphérique...) ; le rapport référent-discours (termes) est donc partiellement encore ambigu;

- ceux qui traitent de ces "pluies " sont de formation diversifiée ; ils ne constituent pas une " communauté » à proprement parler — d'autant que leur engagement s'appuie sur des prémisses différents et s'oriente vers des objectifs plus ou moins divergents ;

- une des difficultés majeures pour établir la terminologie des dépôts acides réside dans le partage des termes : bon nombre d'entre eux préservent leur sens original mais sont d'usage fréquent dans les "pluies acides " qui deviennent alors un nouveau champ d'application - tandis que d'autres acquièrent un sens nouveau. Cela suffit-il à créer un "domaine "? La question est sensible car elle sous-tend la recherche (sélection des documents ; saisie des néologismes ; établissement des rapports entre les notions...). Elle se pose en général dès qu'on a affaire à une nouvelle technique, à un nouveau problème ; la robotique, le Sida sont-ils des "domaines"?

\section{POUR UNE SOCIO-TERMINOLOGIE}

Dans ce qui précède, on a eu l'occasion de s'attarder sur divers points encore problématiques en terminologie, notamment le choix du corpus, la notion de domaine. Les "pluies acides" permettent aussi de s'interroger sur les bases théoriques de cette terminologie qui prétend être au cœur d'enjeux scientifiques, industriels, commerciaux, linguistiques... Il faut reconnaître qu'elle se base sur des postulats, contestables, oblitérant les conditions, les contraintes qui pèsent sur la formulation, la sélection, la distribution, le rejet et l'acceptation des termes ainsi que sur leur signification. Je rappelle quelquesuns de ces principes, de ces $a$ priori :

- la transparence : à une même notion devraient correspondre des termes de formes apparentées dans diverses langues;

- la biunivocité : à une notion devrait correspondre, dans chaque langue, un seul terme et à un terme donné devrait correspondre une seule signification ;

- la monosémie : chaque terme ne devrait avoir qu'un sens, dans un domaine donné :

- le terme (idéal) est motivé, systématique, source de dérivés potentiels, condensé dans sa forme...

Ces postulats ignorent donc la polysémie, les degrés d'équivalence d'une langue à l'autre, font l'embargo sur la synonymie... Plus gravement, ils occultent les faits suivants :

qu'il n'y a pas de terminologie hors de l'histoire, hors des rapports de force : les sens évoluent, changent comme les réalités qu'ils expriment, par rapport à des acquis, des savoirs antérieurs; les frontières des domaines, on l'a vu, se modifient et permettent des transferts de vocabulaire;

- qu'il n'y a pas de terminologie hors des pratiques sociales que sont les discours, hors de conditions d'énonciation définies : les termes ne sont pas des objets en soi, à rattacher à des définitions coupées de toute pratique scientifique, professionnelle ; 
- qu'il n'y a pas de terminologie hors des forces productives en concurrence, en opposition : les notions n'existent pas avant tout dans des réseaux notionnels abstraits.

Derrière ces a priori se profile le rêve d'une langue limpide, s'exprime un volontarisme idéaliste... qui servent parfois le dirigisme, l'obsession normative de certaines organisations terminologiques, intégrées aux rouages de l'État. Poussée à son extrême, cette vision débouche sur une conception totalitaire de la langue et de son fonctionnement, norme figée, sous contrôle unique, aveugle à l'évolution et à l'interpénétration des savoirs, à la rivalité des écoles, des acteurs sociaux, à la compétition internationale, à la place et au statut réels des sciences et techniques dans une société donnée. En outre, les affirmations théoriques de la terminologie, son rationalisme idéaliste, sa mystique objectiviste sont en contradiction avec sa méthodologie, sa pratique qui doit faire avec les termes en contexte, les ambiguiités définitionnelles, avec des sources documentaires plus ou moins fiables, avec le problème épineux de la classification des "domaines", etc.

Nous croyons que la terminologie, avec ses contradictions, dépend d'une réflexion plus large sur la communication entre spécialistes pris entre l'hyperspécialisation et l'interdisciplinarité, sur la dynamique des "domaines " du savoir et de la production (dialectique de l'intégration et de la division) ; nous croyons aussi qu'elle se doit de se transformer d'urgence en socio-terminologie, pour comprendre la circulation des termes, les réactions aux néologismes, les résistances devant les listes terminologiques officielles, pour permettre de définir et d'évaluer des politiques d'aménagement terminologique... Un terme ne peut pas être vu seulement par rapport à un système (adéquation de la désignation, rattachement à un réseau de notions...) : il est aussi à voir dans son fonctionnement, sur le terrain des contradictions sociales. (Qui utilise quoi ? Qui innove ? Comment: et par qui les termes se diffusent-ils ? Comment s'opèrent les réajustements terminologiques, les reformulations? etc.)

La terminologie ne peut faire la pluie... et le beau temps sur des réalités plurielles. Est-ce là propos acide? Non! Surtout dans le cadre d'un colloque qui envisage enfin la terminologie comme fertilisation et non comme abâtardissement, contamination, détérioration, confusion de la langue... infécondable. Certes il y a peut-être paradoxe à soutenir cette fertilisation par le biais... des "pluies acides"!

Notes

1. L'acidité d'un liquide se mesure conventionnellement par un nombre $\mathrm{pH}$ relié à la proportion d'ions hydrogène. L'échelle des pH va de 1 à 14 (du plus acide au plus basique). L'eau distillée a un pH égal à 7 ; l'eau propre légèrement acidifiée par le gaz carbonique de l'air a un pH de 5,6; en deçà de ce seuil, il y a acidité avec effets sur la faune et la flore aquatiques, etc. On a observé des précipitations très acides de 2,6!

2. De la combinaison de l'oxygène de l'air, de la vapeur d'eau avec les oxydes d'azote $\left(\mathrm{NO}_{\mathrm{x}}\right)$ et le dioxyde de soufre $\left(\mathrm{SO}_{2}\right)$, déversés par la combustion externe des carburants fossiles et les moteurs à combustion interne, résultent des acides nitriques $\left(\mathrm{HNO}_{\mathrm{x}}\right)$ et des acides sulfuriques $\left(\mathrm{H}_{2} \mathrm{SO}_{4}\right)$.

3. Recherche trilingue : français, anglais et italien 